\title{
An Operating Model for the Integrated Pest Management of Great Lakes Sea Lampreys
}

\author{
Michael L. Jones ${ }^{1, *}$, Brian J. Irwin ${ }^{1}$, Gretchen J. A. Hansen ${ }^{1,2}$, Heather A. Dawson ${ }^{1,3}$, Andrew J. \\ Treble $^{1,4}$, Weihai Liu ${ }^{1}$, Wenjing Dai ${ }^{1,5}$ and James R. Bence ${ }^{1}$ \\ ${ }^{I}$ Quantitative Fisheries Center, Department of Fisheries and Wildlife, 13 Natural Resources Building, Michigan State \\ University, East Lansing, MI 48824, USA \\ ${ }^{2}$ Current address: Center for Limnology, University of Wisconsin-Madison, 680 North Park Street, Madison, WI 53706, \\ USA \\ ${ }^{3}$ Current address: U.S. Fish and Wildlife Service, Marquette Biological Station, 3090 Wright Street, Marquette, MI \\ 49855, USA \\ ${ }^{4}$ Current address: Department of Fisheries and Oceans, Sea Lamprey Control Centre, 1 Canal Drive, Sault Ste. Marie, \\ ON P6A 6W4, Canada \\ ${ }^{5}$ Current address: Integrated Healthcare Solutions, 13925 Ballantyne Corporate Place, Charlotte, NC 28277, USA
}

\begin{abstract}
Models of entire managed systems, known as operating models or management strategy evaluation (MSE) models, have been developed in recent years to more fully account for uncertainty in multiple steps of fishery management. Here we describe an operating model of sea lamprey management in the Great Lakes and use the model to compare alternative management strategies for sea lamprey control in Lake Michigan. Control of sea lampreys is mainly achieved through the application of chemical lampricides that target stream-dwelling larvae before they become parasites. The operating model simulated uncertainty due to process variation in larval population dynamics, the accuracy of population assessments used to direct selection of areas to be chemically treated, and the effectiveness of these treatments. We used the operating model to compare the performance of stream selection strategies that either rely on assessments to direct chemical treatments or eliminate the assessment process altogether by relying on prior but uncertain knowledge of stream-level sea lamprey growth rates to specify a fixed schedule for chemical treatments. The fixed schedule strategy led to a modest improvement in expected suppression of parasitic sea lamprey abundance over the assessment-based strategy so long as assessment cost savings were allocated to chemical treatment when assessment was not used to select streams for treatment. We also evaluated the sensitivity of the assessment-based strategy to differing but plausible levels of assessment uncertainty. A moderate reduction in assessment uncertainty led to a large increase in suppression of parasitic sea lamprey abundance for the assessment-based selection strategy, emphasizing the importance of both accurately measuring and reducing assessment uncertainty.
\end{abstract}

\section{INTRODUCTION}

Fishery management can benefit greatly from forecasts of the consequences of alternative management strategies that properly account for system uncertainty [1]. Many forms of uncertainty can affect decision making [2, 3], including uncertainty about ecological processes that govern population dynamics (process uncertainty), our inability to observe systems without error (measurement uncertainty), and the "disconnect" between an intended management action and what actually happens in the real world (implementation uncertainty). Until recently, technical challenges prevented explicitly considering these multiple sources of uncertainty for a

*Address correspondence to this author at the Quantitative Fisheries Center, Department of Fisheries and Wildlife, 13 Natural Resources Building, Michigan State University, East Lansing, MI 48824, USA; 517-432-0465; Fax: 517-432-1699; E-mail: jonesm30@msu.edu particular fishery simultaneously. During the past 20 years, however, fisheries scientists, particularly in southern Africa, Australia, and in association with the International Whaling Commission, have successfully developed tools that take into account each of these types of uncertainties and they have been used to evaluate alternative fishery management strategies [4-6]. Their approach entails developing an "operating model" that includes three components: a system model of the fish stock and its associated fishery, an observation model that describes what information can be obtained about the fish stock and the fishery, and a management model that describes how management actions are implemented and their effects on the fishery. In Australia, this approach has been termed Management Strategy Evaluation, or MSE [7]; MSE-style analyses of commercial fishery harvest systems are now widely viewed as a valuable, perhaps even essential, tool for fishery management, especially since the FAO Technical Consultation on the Precautionary Approach to Capture Fisheries [6, 8-10]. 
Fishery management is not limited to capture fisheries, however, and uncertainty exists in other aspects of fishery management [11-13]. In the Laurentian Great Lakes, a critical management challenge is cost-effective control of the exotic sea lamprey Petromyzon marinus, which invaded the upper Great Lakes in the 1930s and devastated fish communities [14]. Today, sea lamprey populations are controlled through a bi-national integrated pest management program led by the Great Lakes Fishery Commission (GLFC). The integrated program reduces parasitic sea lamprey production by targeting larval habitats with chemical lampricides [15], as well as employing various measures to reduce the reproductive success of adult sea lampreys as they return to Great Lakes tributary streams to spawn [16-18]. Each year the GLFC decides how to allocate limited pest control resources; for the lampricide program they have developed a management strategy that utilizes assessment information on larval sea lamprey abundance and distribution in streams to select a set of streams for lampricide treatment each year [19, 20]. The performance of this management strategy can be affected by process, assessment, and implementation uncertainty, but until recently these uncertainties have been largely ignored. In this paper, we describe an operating model for sea lamprey control that incorporates these uncertainties and our use of the model to explore alternative assessment strategies for the stream selection process for lampricide control.

\section{BACKGROUND ON THE MANAGEMENT SYSTEM}

Adult sea lampreys migrate into streams tributary to the Great Lakes during spring and spawn from late May through July. After hatching, larval sea lampreys drift downstream, burrow in soft substrates, and become filter-feeding ammocoetes. After 3-6 years spent in these habitats, most larvae begin metamorphosis, at which time they develop the feeding apparatus required for the parasitic life stage. Metamorphosed sea lampreys (called transformers during migration) migrate downstream to the lake in fall or spring where they feed on host teleost fishes for 12-18 months before maturing and completing their semelparous life cycle.

Most sea lamprey control is achieved through the periodic treatment of lotic and lentic habitats with the lampricides TFM (3-triflouromethyl-4-nitrophenol) and Bayluscide (2'5-dichloro-4'-nitrosalicylanilide), respectively. For a given budget the maximum level of control will be achieved if lampricide applications are directed to streams with the greatest abundance of larval sea lampreys expected to metamorphose in a given year relative to the cost of lampricide treatment for that area [21]. An assessment program is required to determine which streams contain the greatest number of larval sea lampreys expected to metamorphose in each year $[19,20]$. The current larval assessment program used to prioritize streams for treatment is costly relative to the overall funds available for pest management; in recent years, larval assessment has accounted for one quarter to one third of the total sea lamprey management budget. Despite the high level of investment by the GLFC, the population estimates obtained from this assessment program are highly uncertain $[22,23]$. Furthermore, the magnitude of population reduction in a stream treated with lampricides, while often assumed to be $95 \%$ or greater, can vary considerably across locations and over time, in part depending on environmental conditions (e.g., stream discharge) at the time of treatment.

Sea lamprey control is carried out in the North American Great Lakes to allow both wild and stocked teleost fishes, especially trout and salmon, to survive in sufficient numbers to contribute to a viable fishery and spawning population. Thus the primary objective of sea lamprey control is to achieve target levels of abundance of larger, older host fishes (mainly salmonines). In our analysis, however, we use the forecasted abundance of adult sea lampreys as our measure of performance for management strategies. Because sea lamprey abundance is far below and host abundance is far above the historical levels observed prior to the implementation of the control program, we can reasonably assume that sea lamprey attacks on hosts and thus host survival will be proportional to sea lamprey abundance over the range of abundances relevant to this analysis - moderate numbers of additional sea lamprey are unlikely to experience significant effects of exploitative competition [24].

Here we describe how we incorporated uncertainties about sea lamprey population dynamics, assessment uncertainty, and implementation error into an operating model. We used the model to compare the performance of management strategies that either rely on larval assessment to direct selection of streams for chemical treatment or instead operate on a fixed-treatment schedule, thus eliminating the need for larval assessment. We also considered the influence of assessment uncertainty on this comparison. Surprisingly, there are few other evaluations where alternative choices about how to allocate budgetary resources between population assessment and other management actions have been assessed [25].

\section{THE OPERATING MODEL}

\section{Overview}

We developed a stochastic age-structured population model to forecast changes in future sea lamprey abundance for each of the Laurentian Great Lakes resulting from implementation of a particular pest management strategy. The model includes the full life cycle for the population of sea lampreys occupying the Great Lakes and tributary streams and forecasts changes in abundance over a 100-year time horizon. The model represents the sea lamprey life cycle as having the following stages: larval, transformer or metamorphosing, parasitic, and spawning-phase (Fig. 1). To simulate control of sea lamprey production through application of lampricides, the model represents larval populations at the level of individual stream reaches that are the spatial units of chemical control. Spawning-phase sea lampreys are allocated to stream reaches based on stream size and larval abundance, then produce age- 0 recruits based on a stock-recruitment function, and subsequently die. The operating model also allows stream-level manipulation of adult spawning success to simulate adult control tactics such as trapping of migrating adults or barriers to restrict access to spawning habitats. However, we will not be considering management strategies that include adult control in this analysis. Existing physical barriers are implicitly included in the model because only stream habitats downstream of such barriers are included as candidate stream reaches for lampricide control. 


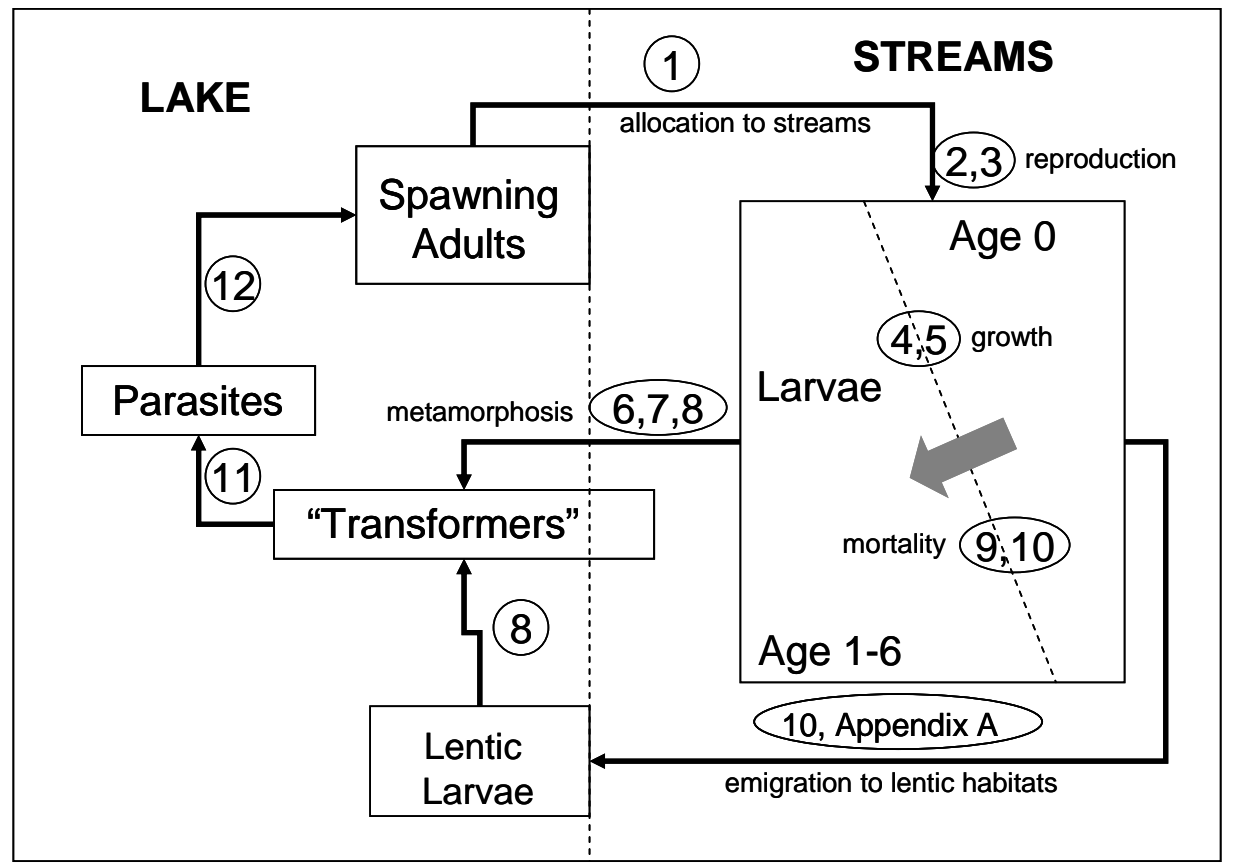

Fig. (1). A sea lamprey life history diagram, showing the movement from the lake to streams during the adult phase and the return to the lake as parasites. Transformers refers to sea lamprey individuals that have recently completed metamorphosis to the parasitic life stage. The numbers reference model equations in the text for the various demographic processes. Also depicted is emigration of age- 0 sea lamprey larvae to lentic habitats, as described in Appendix A.

Because larval sea lampreys typically remain in streams for multiple years, the age- and length- structured larval population (ages 0 through 6 years) in each stream is updated annually to account for natural mortality, removals due to lampricide treatment, and losses due to metamorphosis to the parasitic life stage. The larval sea lampreys that complete metamorphosis and are not removed via lampricide control are added to the parasitic population for the lake. This cohort of parasites becomes the spawning-phase population in the following year after losses due to parasitic-phase natural mortality. In addition to the larval populations in stream habitats, the model also represents alternative larval habitat areas (Appendix A). The model includes uncertainty in biological processes (recruitment and growth), assessment of larval populations, and implementation of lampricide control. Definitions for all model parameters are provided in Table 1.

The complete set of sea lamprey-producing streams for each Great Lake was explicitly represented, following the approach used by the GLFC (Gavin Christie, GLFC, Ann Arbor, MI, personal communication) in which large streams are divided into reaches for which independent lampricide treatment decisions can be made. We relied on an existing database (known as the Empiric Stream Treatment Ranking [ESTR] database, used by the GLFC to select streams for lampricide treatment) as a source of physical (stream lengths, widths, etc), biological (larval growth rates and growing season lengths), and economic (treatment costs) information for streams [19]. Below we describe the details of the operating model as it applies to each of the Great Lakes, and note areas where the model has stream-, lake-, or region-specific parameter values. The application of the model presented in this paper, however, is for Lake Michigan only (Fig. 2).

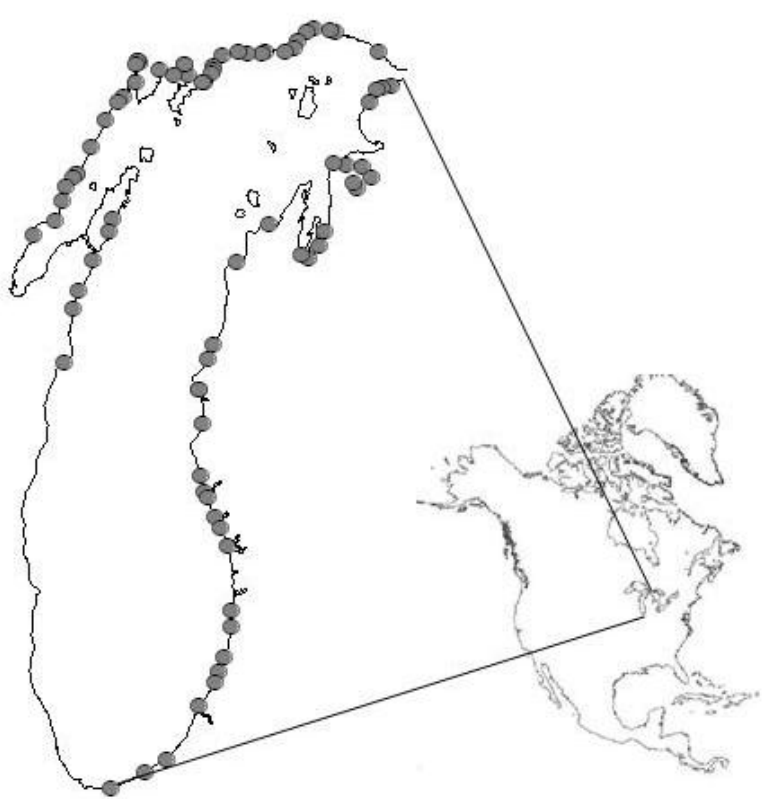

Fig. (2). A map of Lake Michigan showing (solid circles) the location of tributary streams and rivers that are regularly infested with sea lampreys and treated for control.

\section{Biological Model}

Adult sea lampreys do not home to natal streams [26], therefore spawning-phase sea lampreys were allocated to stream reaches based on two rules: $i$ ) the drainage area of the stream, as streams with the greatest discharge have been found to accommodate the largest number of spawning- 
Table 1. Parameters, Their Assumed Values, and State Variables Used in the Operating Model for Sea Lamprey

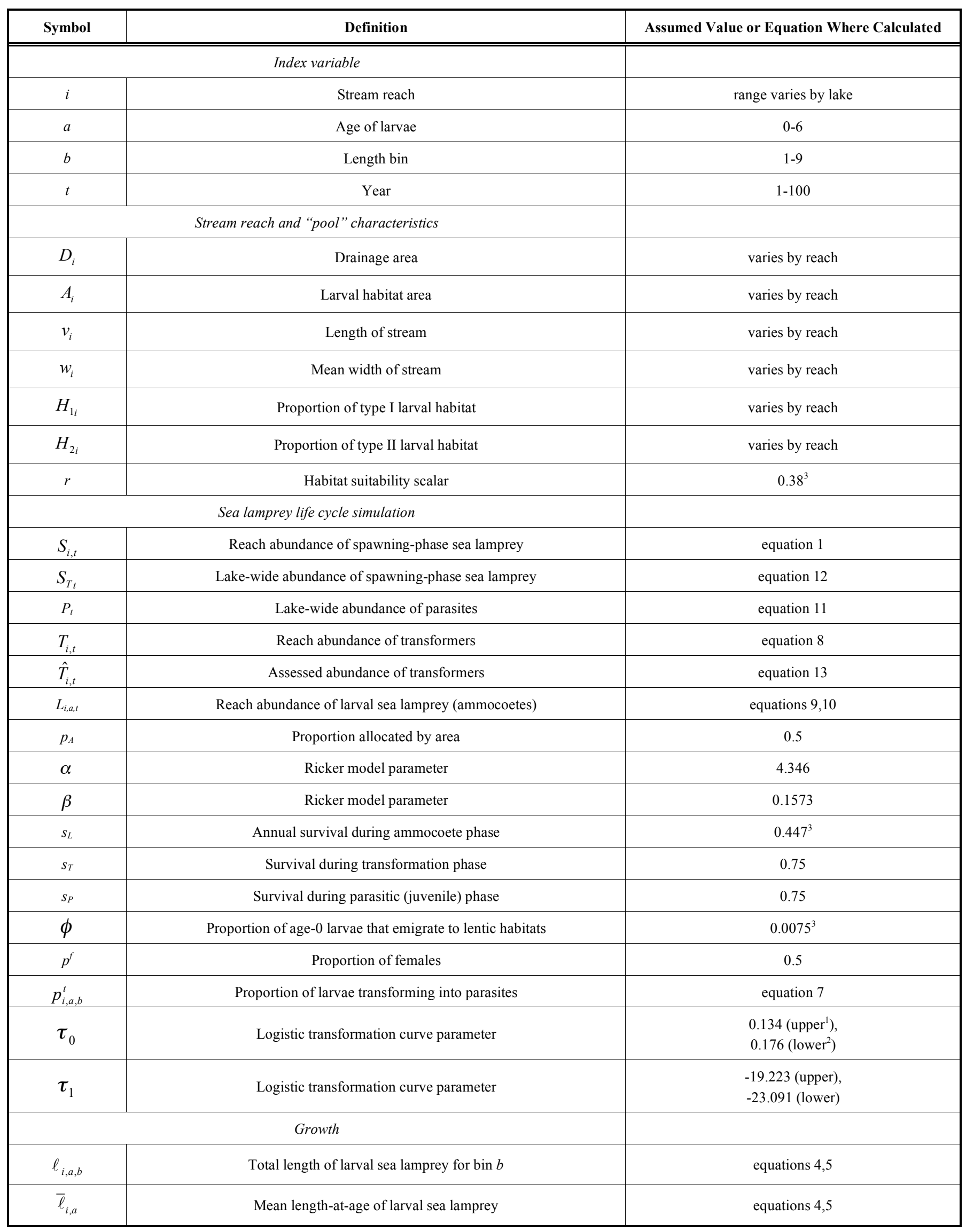


Table 1. contd...

\begin{tabular}{|c|c|c|}
\hline Symbol & Definition & Assumed Value or Equation Where Calculated \\
\hline$d_{i}$ & Duration of growing season (number of days) & varies by reach \\
\hline$\ell_{0}$ & Initial von Bertalanffy length in millimeters & 20 \\
\hline$\ell_{\infty b}$ & Size bin-specific von Bertalanffy asymptotic length (mm) & equation 4 \\
\hline$\varepsilon_{b}$ & Deviations about mean length & range is -2 to $+2 \mathrm{SD}$ \\
\hline$\dot{p}_{i, a, b}$ & Proportion of larvae at age in a length bin & see text \\
\hline$C_{i}$ & Cost of chemical control (dollars) & varies by reach \\
\hline$\hat{E}_{i}$ & Anticipated treatment effectiveness & varies by reach \\
\hline $\mathrm{CV}_{T}$ & Coefficient of variation in assessment error & 0.99 or 1.71 \\
\hline \multicolumn{3}{|c|}{ Uncertainty terms } \\
\hline$\varepsilon_{i, t}^{r}$ & Variance of process error in larval recruitment to age-1 & $\sim \mathrm{N}(0,3.39)$ \\
\hline
\end{tabular}

1 "upper" refers to the upper Great Lakes - Superior, Michigan, and Huron

2 "lower" refers to the lower Great Lakes - Erie and Ontario

${ }^{3}$ Lake Michigan values; different values are used for the other Great Lakes

phase sea lampreys if other environmental factors are favorable $[27,28]$, and $i i)$ the abundance of larvae in each stream reach, as sea lampreys have been shown to be attracted to a migratory pheromone released by stream-dwelling ammocoetes [29]. Each year, lake-wide spawning-phase sea lampreys were divided into those that migrate based on drainage area and those that migrate based on the abundance of larvae in each reach. Then, the number of spawning-phase sea lampreys allocated to a given reach was assumed to be positively related to both the drainage area and larval abundance, relative to other stream reaches:

$S_{i, t}=S_{T_{t}} p_{A} \frac{D_{i}}{\sum D_{i}}+S_{T t}\left(1-p_{A}\right) \frac{L_{i, t}}{\sum L_{i, t}}$

In the simulations used in this paper, the two mechanisms were given equal weight (i.e., $p_{A}=0.5$ ).

Recruitment of larvae was calculated from the abundance of spawning-phase sea lampreys in each stream reach using a stochastic Ricker stock-recruitment function [30, 31].
$L_{i, 0, t}=\alpha S_{i, t} p^{f} e^{-\beta \frac{S_{i, t} p^{f}}{A_{i}}+\varepsilon_{i, t}^{r}} / S_{L}$

The density-dependent term of the recruitment function depended on the area of suitable larval habitat in the stream reach, which was obtained from surveys of the length, average width and proportion of wetted area comprising two types of habitat used by larval sea lampreys: preferred (Type 1) and acceptable (Type 2). We calculated a weighted area of suitable larval habitat from:

$A_{i}=v_{i} w_{i}\left(H_{1 i}+H_{2 i} r\right)$

based on a conversion factor $(r)$ derived from the observed ratios of sea lamprey density between these two habitat types [20]. We used lake-specific conversions based on empirical evidence of differences in this ratio among lakes (M.L. Jones, unpublished data). The other recruitment parameters for equation 2 were estimated using a meta-analysis of data from 90 stream-years of sea lamprey stock-recruitment data [31]. We chose the Ricker function to model recruitment 
because we observed reduced recruitment at high abundances of spawning-phase sea lamprey in these data. We assumed that inter-annual density-independent recruitment variation was not correlated among streams (no common "year effects") based on empirical analyses [31]. The empirical model related abundance of spawning-phase sea lampreys to observed age- 1 abundance the following year, so the number of age- 0 sea lampreys in year $t$ was calculated from predicted age- 1 abundance in year $t+1$ using an annual survival rate $\left(s_{L}\right)$ assumed constant among larval ages and stream reaches.

Sea lamprey metamorphosis to the parasitic stage is governed at least in part by length [32], so we modeled the larval population as both length- and age-structured. Our approach was to consider the population as consisting of a set of different growth types, with all individuals of a given growth type being represented by a common length at age. These growth types are defined by the length bins individuals started in at age-1, and subsequent length-at-age for larval sea lamprey in a length bin $(b)$ follows a von Bertalanffy growth model:

$\ell_{i, a+1, b}=\ell_{i, a, b}+\Delta \ell_{i, a, b}$

where $\Delta \ell_{i, a, b}=\left(\ell_{\infty b}-\ell_{i, a, b}\right)\left(1-e^{\left(-\kappa_{i} a d_{i}\right)}\right)$

Nine length bins were defined, and the initial lengths and allocation of proportions of larvae to each bin were set to approximate a presumed normal distribution. The lengths associated with each length bin for age-1 were set to range from -2.0 to +2.0 standard deviations around the mean length at age- 1 in increments of 0.5 for $b=1$ to 9 . Mean age- 1 length was projected from a mean age- 0 length, $\bar{\ell}_{0}$, of 20 $\mathrm{mm}$ based on equation 4 , where the mean age- 0 length plays the role of $\ell_{i, a, b}$, and the assumed coefficient of variation in length at age-1, $\mathrm{CV}_{\ell}$, was 0.08 . Thus:

$\ell_{i, a=1, b}=\bar{\ell}_{i, a=1}\left(1+\mathrm{CV}_{\ell} \varepsilon_{b}\right)$

where $\varepsilon_{b}$ varies from -2.0 to +2.0 in increments of 0.5 . The same distribution and calculations were applied to generate bin-specific asymptotic length parameters, $\ell_{\infty b}$, where the mean value, $\bar{\ell}_{\infty}$, was estimated from growth data for larval sea lamprey (using mark-recapture data from Treble [33]). The remaining stream-specific parameterization of this growth model is explained in Appendix B. The true streamspecific parameters for growth are not known, so we allowed for this uncertainty in our simulations. We achieved this by using the among-stream variability in two growth rate parameters (average daily growth and growing season length) for streams reported in the ESTR database as an empirical sample of possible growth rates for each stream. We accounted for expected geographical variation in growth rates among streams tributary to a particular lake by grouping streams regionally according to spatial patterns of growth variation within a lake basin. For Lake Michigan, we assigned each stream to one of three groups: Indiana and the southern lower peninsula of Michigan; Wisconsin and the northern lower peninsula of Michigan; and the upper peninsula of Michigan. We assigned actual growth rate parameters for individual streams by randomly choosing a pair of values (sampling with replacement) for average daily growth rate and growing season length from the set of candidate pairs for the group to which the stream belonged for each simulation.

For age-1 larval sea lamprey in all stream reaches, the proportions of the total larval population falling into each bin, $\dot{p}_{i, a=1, b}$, were set to $0.04,0.07,0.12,0.17,0.20,0.17$, $0.12,0.07,0.04$ for bins 1 to 9 respectively to approximate the desired normal distribution. For subsequent ages, $\dot{p}_{i, a, b}$ was updated to reflect losses within a bin due to metamorphosis:

$$
\dot{p}_{i, a+1, b}=\frac{\dot{p}_{i, a, b}\left(1-p_{i, a, b}^{t}\right)}{\sum_{b=1}^{9} \dot{p}_{i, a, b}\left(1-p_{i, a, b}^{t}\right)}
$$

The probability of metamorphosis for an individual larval lamprey depended on its length and was calculated for ages2 and older:

$$
p_{i, a, b}^{t}=\frac{e^{\left(\tau_{0}+\tau_{1} \ell_{i, a, b}\right)}}{\left(1+e^{\left(\tau_{0}+\tau_{1} \ell_{i, a, b}\right)}\right)}
$$

The parameters for equation 7 were assumed to differ between the upper (Superior, Michigan, and Huron) and lower (Erie and Ontario) Great Lakes [23] (see Table 1). Note that the proportions $\dot{p}_{i, a, b}$ are not influenced by mortality because this rate was assumed to be the same for each length bin. The number of transformers (potential parasites) in each stream reach was then calculated across ages 2 through 6 and all 9 length bins within an age group by:

$T_{i, t}=\sum_{a=2}^{6}\left(L_{i, a, t} \sum_{b=1}^{9}\left(\dot{p}_{i, a, b} p_{i, a, b}^{t}\right)\right)$

A similar sequence of calculations (equations 4-8) was used to model the population dynamics of larval sea lampreys in lentic habitats and areas not susceptible to control actions (Appendix A).

The larval population in each stream reach was updated annually by adding new recruits to age 0 (equation 2 ) and by subtracting losses due to natural mortality, chemical control, and metamorphosis to the parasitic stage:

$$
\begin{aligned}
& L_{i, a+1, t+1}=L_{i, a, t}\left(1-\sum_{b=1}^{9}\left(\dot{p}_{i, a, b} p_{i, a, b}^{t}\right)\right)\left(1-m_{i, t}\right) s_{L} \\
& a=1,2 \ldots .5
\end{aligned}
$$

For age-1 larvae there is an additional loss term, due to outmigration of age 0 larvae to lentic areas (see Appendix A) and there are no losses due to metamorphosis: 
Table 2. Summary of the Management Strategies and Associated Levels of Two Sources of Uncertainty Implemented Using the Operating Model for Lake Michigan

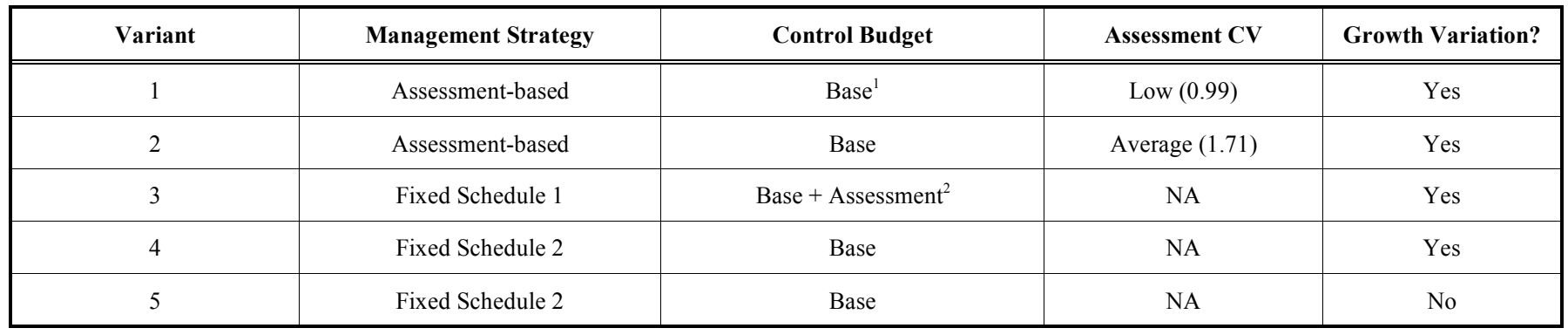

${ }^{1}$ Base control budget for Lake Michigan was \$2.027 million USD

${ }^{2}$ Assessment savings of $\$ 345000$ USD were added to the base control budget

$\mathrm{NA}=$ not applicable, because assessment information was not used to rank streams

$$
L_{i, 1, t+1}=L_{i, 0, t}\left(1-m_{i, t}\right) s_{L}(1-\phi)
$$

These equations implicitly assume that the few larvae that survive to age- 6 and do not transform at that age will die and not reach older ages.

The annual number of parasites leaving larval habitat areas was calculated from the previous year's population of transformers that survived treatment in treated areas (called residuals; see Chemical treatment below) combined with those present in untreated areas $(j$ in equation 11$)$ :

$$
P_{t}=\left(\sum_{i} T_{i, t-1}\left(1-m_{t}\right)+\sum_{j} T_{j, t-1}\right) s_{T}
$$

Finally, the total lake-wide population of adult sea lamprey in year $t+1$ was calculated from the number of parasites surviving in year $t$ :

$S_{T_{t+1}}=S_{P} P_{t}$

\section{Management Strategies}

We used the sea lamprey operating model to compare the performance of five management strategy variants based on two basic strategies for stream selection. We simulated the chemical treatment of streams following either an assessment-based rule generated from a transformer kill-per-dollar estimate or a fixed-treatment schedule in which the number of years between treatments for each treatable area was predetermined. For the assessment-based strategy of stream selection, we evaluated two variants with different levels of assessment uncertainty. For the fixed-treatment schedule we considered two budget options: in one variant we assumed savings from the absence of larval assessment were used to increase the chemical treatment budget (i.e., treat more streams per year); in the other variant we assumed the chemical treatment budget remained the same as for the assessment-based strategy. Finally, we also considered an additional variant in which growth uncertainty was eliminated, speculating that the assessment savings could be used to reduce this source of uncertainty. Although it is likely that some of this growth uncertainty is due to process variation which is probably not reducible, we chose to set growth uncertainty to zero for this variant to assess the maximum benefit that could be derived from reducing this source of uncertainty, analogous to an Expected Value of Perfect Information calculation [2], Thus five different management strategy variants were considered (Table 2).

\section{Assessment-Based Strategy for Stream Ranking}

To select stream reaches for chemical treatment based on assessment information, we simulated the annual stream ranking procedure used since 1995 in the sea lamprey control program [19]. Estimated transformer abundance (i.e., population assessment) was calculated as a function of the "true" transformer abundance:

$\hat{T}_{i, t}=T_{i, t} \varepsilon_{i, t}^{\hat{T}}$

where $\varepsilon_{i, t}^{\hat{T}}$ was drawn from a gamma distribution with a mean of 1 and a CV of either 0.99 (the "low" assessment error) or 1.71 (the "average" assessment error). The two levels of assessment error represent the minimum and average CVs resulting from Monte Carlo simulations performed by Steeves [22] using assessment data from nine Great Lakes streams.

In each year of a simulation, areas available for treatment were ranked in order of treatment priority based on their relative estimated cost-effectiveness of treatment (i.e., ranked to maximize the estimated number of transformers killed per dollar of treatment cost):

$$
\frac{\hat{T}_{i, t} \hat{E}_{i}}{C_{i}}
$$

As noted earlier, this approach will yield the maximum level of suppression per dollar spent when the variables in equation 14 are known without error [21]. For ranking purposes, we used the treatment effectiveness values $\left(\hat{E}_{i}\right)$ assumed by the GLFC in their ranking procedures. These values varied among stream reaches and ranged from 0.75 to 0.99 (proportion killed) but were constant over time. The anticipated treatment cost and effectiveness values were derived from data on previous treatments and expert judgment. We assumed treatment effectiveness in lentic habitats was 0.75 based on recorded treatment assessments for such areas. 
Table 3. The Number of Stream Reaches in Lake Michigan with Cycle Lengths of 4-9 Years for Each Fixed Treatment Schedule

\begin{tabular}{|c|c|c|}
\hline Cycle Length & Fixed Schedule 1 & Fixed Schedule 2 \\
\hline \hline 4 years & 14 & 14 \\
\hline 5 years & 37 & 67 \\
\hline 6 years & 38 & 11 \\
\hline 7 years & 3 & 0 \\
\hline 8 years & 1 & 1 \\
\hline 9 years & 0 & 14 \\
\hline
\end{tabular}

Chemical control costs for each stream reach were obtained from recent data on lampricide costs. Control costs for lentic habitat units were fixed at $\$ 5000 \mathrm{ha}^{-1}$ based on recent treatments of multiple lentic units within the St. Marys River, Lake Huron. In general, stream reaches and treatable lentic habitats with high densities of large ammocoetes (i.e., those likely to metamorphose into parasites within a year) and modest treatment costs were ranked highest. For the Lake Michigan simulations reported here, we used an annual control budget of $\$ 2.027$ million for all simulations and all years when the assessment-based approach was used to rank treatment areas. Each year, reaches were treated in rank order, from highest to lowest, until the budget apportioned to lampricide control was exhausted or until remaining funds were insufficient to treat any remaining locations.

\section{Fixed-Treatment Schedule Strategy}

We compared the assessment-based strategy to an alternative strategy that used a fixed-treatment schedule to apply chemical control to Lake Michigan sea lamprey producing streams. The fixed-treatment schedules did not rely on assessment information to rank streams for treatment, but rather used prior information on stream-specific larval growth rates to order streams according to the rate at which larval cohorts would reach a size where metamorphosis was likely. Streams with faster growth rates were treated more frequently, subject to budgetary constraints on the total number of streams that could be treated each year. We considered two fixed-treatment variants with contrasting budgets for chemical control. In 2007, \$2.9 million was spent on larval assessment in the Great Lakes basin, of which $\$ 1.38$ million was used for surveys aimed at ranking streams for treatment (G. Christie, GLFC, Ann Arbor, MI, personal communication). Lake Michigan contains approximately $25 \%$ of all treated stream reaches in the Great Lakes basin, so we assumed for the simulations reported here that $25 \%$ of the budget for stream ranking assessment (\$345 000) would have been used for the assessment of Lake Michigan streams. The control budget used for generating the first treatment schedule (Fixed Schedule 1) was the sum of the base control budget ( $\$ 2.027$ million) and the reallocated assessment funds for a total control program expenditure of $\$ 2.372$ million. The second treatment schedule (Fixed Schedule 2) was generated under the assumption that additional funds would not be available to augment chemical control even if assessment for ranking streams was elimi- nated, and thus the control budget for this variant remained at the base amount of $\$ 2.027$ million. The variant in which we assumed growth uncertainty was eliminated was only applied to Fixed Schedule 2, based on the premise that savings from assessment would be applied to reducing growth uncertainty rather than towards increased chemical control.

Fixed-treatment schedules were designed by assigning a cycle length representing the number of years between treatments to each treatment unit (stream reach or lentic habitat unit). Stream reaches with the highest estimated annual growth rates and lentic habitat units with the highest levels of recruitment were assigned the shortest cycle lengths. The overall annual budget available for treatment, as well as the costs of treating all treatment units in Lake Michigan, determined the range of cycle lengths; on average cycle lengths were longer for Fixed Schedule 2 than Fixed Schedule 1 because the smaller budget for the former allowed fewer treatment units to be on the schedule in any particular year (Table 3). We used an iterative and by necessity somewhat ad hoc procedure to determine the cycle lengths for all stream reaches by first ordering reaches from fastest to slowest growth rates, and then varying the cycle lengths for individual streams subject to the constraints imposed by the annual treatment budget and that reaches with faster growth rates never had a longer cycle length than a stream with a slower growth rate. We did not have growth rate data for the lentic habitat units, so these areas were assigned cycle lengths ranging from 4 (highest recruitment areas) to 13 (lowest recruitment areas) years, for both budget variants. These lentic habitat units comprise a small proportion of the sea lamprey larval habitat in Lake Michigan (assumed to total less than $4 \%$ ) that is available for treatment and are also believed to support lower densities of larvae than favorable stream habitats. After assigning a cycle length to each area available for treatment, we generated fixed schedules that assigned areas to actual simulation years and which minimized the annual variability in treatment budgets. In each iteration and each year, all areas scheduled for treatment were treated, and the budget required to do so was recorded. In the fixed-treatment variants, the actual amount expended on control varied from year to year based on which areas were due for treatment, but with the overall average annual expenditure set by the available budget.

\section{Chemical Treatment}

Once an area is selected for chemical treatment, the actual treatment effectiveness is subject to a variety of influ- 


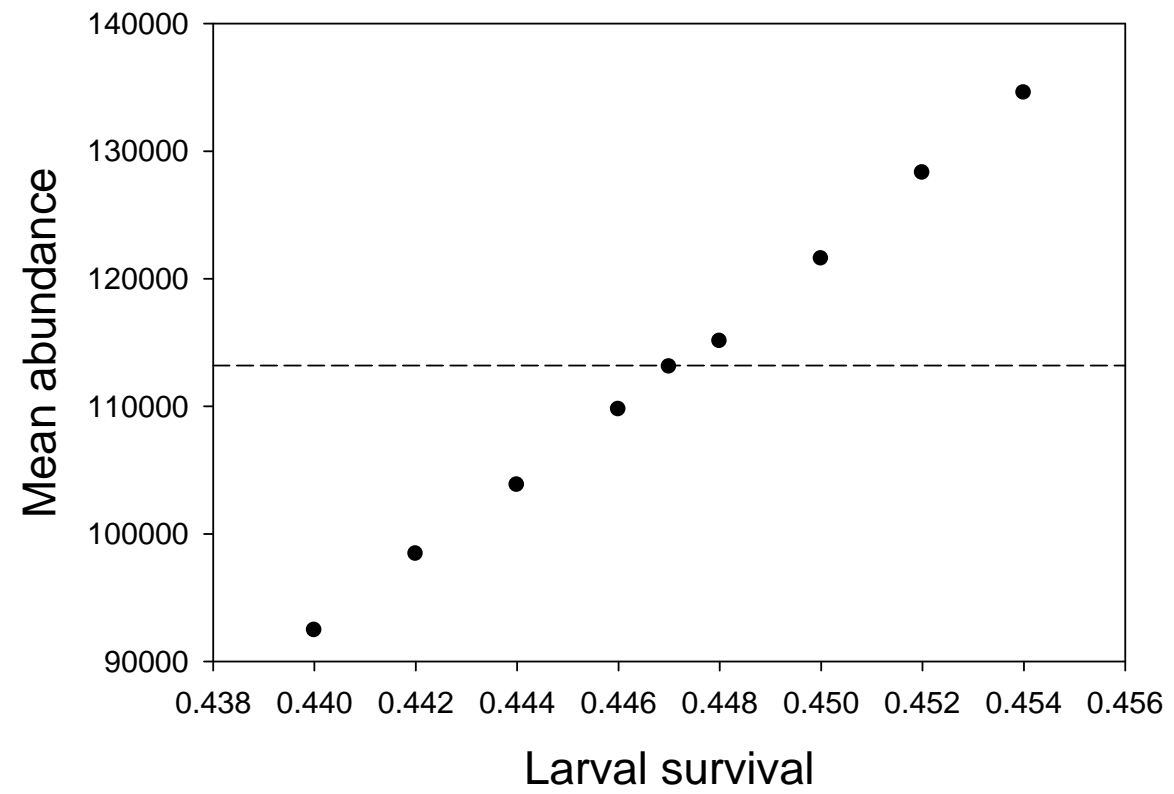

Fig. (3). Calibration results showing mean abundance of spawning-phase sea lamprey averaged across the final 10 years of the 100 -year simulations and then averaged over simulations across a range of larval survival rates. Horizontal reference line shows calibration target abundance value of 113200 .

ences (e.g., discharge, changes in $\mathrm{pH}$ ), although most stream treatments result in levels of larval mortality exceeding $90 \%$. Therefore, if a simulated stream reach was selected for TFM treatment, the entire larval population in that area was reduced based on drawing an estimate of mortality (i.e., treatment effectiveness; $m_{i, t}$, equations 9-11) from a highlyskewed beta distribution that produced an average treatment mortality near 93\% with a CV around 0.10 . Little is known about the variability of treatment effectiveness in lentic habitats, although treatment of these habitats is generally considered less effective than lotic control. We assumed that actual mortality due to chemical treatment in a lentic habitat unit would vary about a mean $75 \%$ according to a normal distribution with a $\mathrm{CV}$ of 0.10 . Treatment mortality was applied to all larvae present in the treated area, regardless of age or length (equations 9-10). For each simulation, the applied control mortality values were drawn for all locations regardless of rank status for all years, but only applied to streams that were selected for treatment. This way, treatment effectiveness values related to a single location may vary over time, but treatment effectiveness values did not vary across simulations for cases where the same location was treated in the same year.

\section{Model Calibration and Simulations}

Prior to running the simulations, the operating model was calibrated based on stream data for Lake Michigan, as well as information on both recent control expenditures (7-year mean of recent annual lampricide budgets, 1998-2004) and recent observed values of abundance for spawning-phase sea lampreys (7-year mean, 2000-2006), all provided by the GLFC. The calculations of target calibration abundance and control budget values were offset by a two-year lag to approximate an expected delay between treatments targeting larval lamprey and measures of the adult population. The goal of the calibration process was for the simulation model to approximate spawning-phase sea lamprey abundances close to recent observations when using control budgets that correspond to actual recent expenditures in Lake Michigan. The calibration budget was the combined costs associated with TFM and treatment staff (effort). Thus, the calibration budget was the same as the base control budget used during the evaluation of both management strategies. Larval survival was the primary adjusted parameter during calibration based upon earlier work [34] and because it is an important demographic parameter for which we presently have very limited information. In addition to the larval survival rate, we adjusted an outflow scalar which determined the movement of age- 0 larvae from streams and the untreated habitat into lentic habitats. This outflow scalar was adjusted so that density of larvae in lentic areas was approximately one quarter of the average density in streams during the calibration runs. This target ratio was based on observed densities from a lentic inventory survey where larval lamprey densities were measured in both natal lotic areas and associated lentic habitats (Mike Steeves, Department of Fisheries and Oceans Canada, unpublished data).

To compare simulated sea lamprey abundance to the calibration target abundance, we defined the equilibrium level as mean abundance of spawning-phase sea lampreys resulting from sustained treatment. For each simulation of the calibration control budget, the simulated abundance of spawning-phase sea lampreys was averaged across the final 10 years. Then, a grand mean was also calculated by averaging across simulations. The larval survival rate was adjusted until the grand mean was approximately equal to the calibration target abundance (Fig. 3). We used 5000 simulations with a 100 year time horizon for calibration. Once the operating model was calibrated to recent targets for Lake Michigan, alternative control variants could then be explored. 


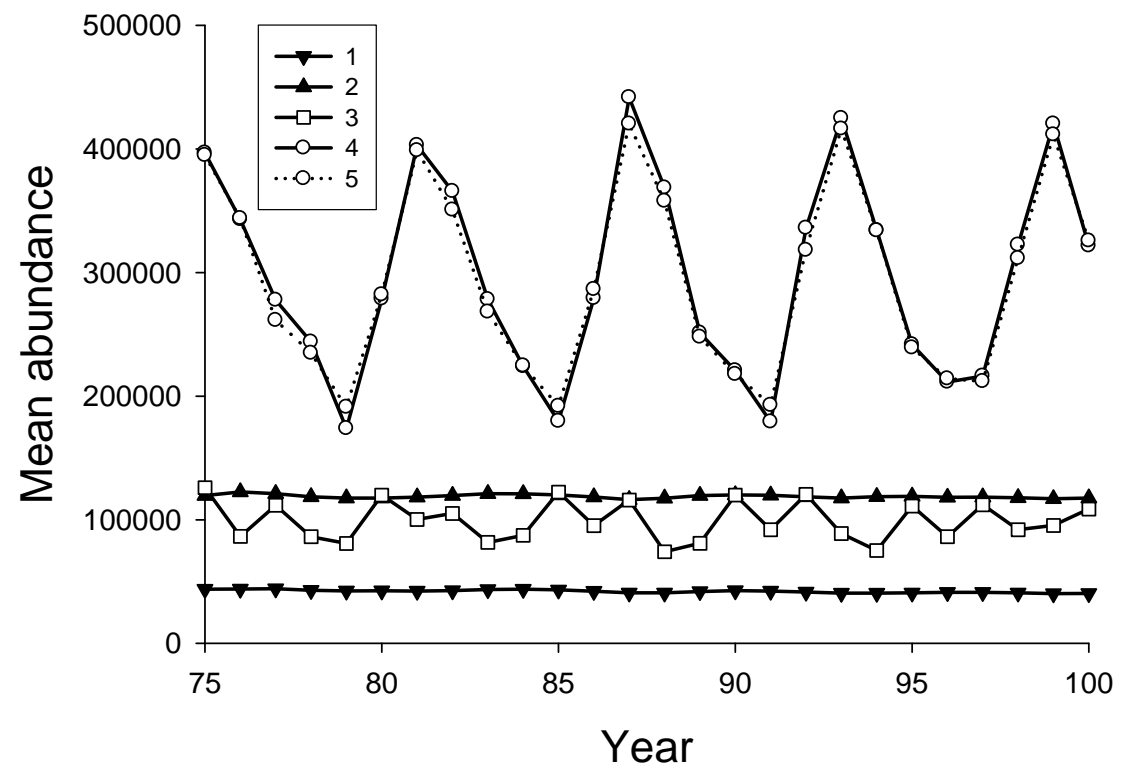

Fig. (4). Mean abundance of spawning-phase sea lampreys across 5000 iterations for years 75-100 of the 100-year simulations for each of the five variants described in Table 2. Management strategy variants are identified in the figure legend as indicated in Table 2 and include assessment-based with either low $(\boldsymbol{\nabla})$ or average uncertainty $(\boldsymbol{\Delta})$, a fixed-treatment schedule with some budgetary resources shifted to control ( $\square$; Fixed Schedule 1), a fixed-treatment schedule without additional control expenditures ( $\circ$, solid line; Fixed Schedule 2), and this same fixed-treatment schedule without growth parameter variability ( $\circ$, dotted line; Fixed Schedule 2).

To compare policies, all simulations were also run for 100 years to allow abundance of spawning-phase sea lampreys to approach a stationary distribution of values prior to the final years of the simulation when model estimates were evaluated. Each policy was repeated 5000 times to account for model uncertainty. For each set of 5000 simulations, the mean abundance of spawning-phase sea lampreys was recorded for the final ten years $(t=91-100)$ and the grand mean was calculated across all simulations. Results for years 75-100 are also presented to display temporal trends (Fig. 4).

\section{RESULTS}

\section{Initialization of the Operating Model and Initial Popula- tion Dynamics}

Each simulation began with an initial population size of 75000 spawning-phase sea lampreys and an age-0 larval density of $1 \mathrm{~m}^{-2}$ based on specified larval habitat areas. These homogeneous initializations are necessary simplifications, given incomplete knowledge of age-specific abundances for sea lamprey in all Lake Michigan tributaries, and result in marked transient population dynamics during the early (first 10-15) years of a simulation. Visual inspection of temporal patterns in projected abundance indicated that stationary conditions were approached after approximately 50 years.

\section{Comparison of Management Strategy Variants}

For each management strategy variant, the treatment expenditure varied annually depending on which areas were actually treated in that year. For each fixed-treatment variant, the mean annual treatment expenditure across all years and simulations was slightly lower than, but by no more than $1.5 \%$, of the budget expected to be available (Table 4). Annual treatment expenditures varied minimally among years for both assessment-based variants and were within $1 \%$ of the target budget.

The model was calibrated using the assessment-based strategy with the average level of uncertainty (Variant 2, Table 2); this variant best represents the status quo for management and the recent history of control on Lake Michigan. The forecasted future abundance of spawning-phase sea lampreys for this variant was 118162 (Table 4), which is within the range of estimates of abundance from adult assessment surveys during the calibration period ( $85800-164$ 700 , unpublished data). The variability among simulations in this forecasted abundance is large ( $\mathrm{SD}=87095$, Table 4), reflecting the high magnitude of process uncertainty for sea lamprey recruitment.

Selecting streams for treatment using a fixed-schedule rule, but with the same overall (treatment and assessment) budget (Variant 3, Table 2), resulted in a lower forecasted mean abundance of spawning-phase sea lampreys than was seen for Variant 2. The difference was small relative to the variability in outcomes among simulations, but in $70 \%$ of cases this variant resulted in lower forecasted abundance than status quo variant (Table 4). If a fixed schedule was used without increasing the treatment budget by an amount equal to assessment savings (Variant 4, Table 2), then the average forecasted spawning-phase sea lamprey abundance was substantially higher, and the simulations almost never resulted in an instance of lower forecasted abundance than the status quo variant (Table 4, last column). As well, this strategy resulted in wide inter-annual variation in forecasted abundance (Fig. 4). For this variant, the majority of stream reaches were treated on a cycle of six years or more (Table 3), longer than the duration of the larval phase in some of these streams. 
Table 4. Summary of Results for Each Management Strategy Variant Described in Table 2

\begin{tabular}{|c|c|c|c|c|c|c|c|c|}
\hline Variant & Management Strategy & $\begin{array}{c}\text { Assessment } \\
\text { Budget } \\
\text { (US \$) }\end{array}$ & \multicolumn{2}{|c|}{$\begin{array}{c}\text { Annual } \\
\text { Treatment Expenditure } \\
\text { (US \$) }\end{array}$} & \multicolumn{2}{|c|}{$\begin{array}{c}\text { Spawning-phase } \\
\text { Sea Lamprey }\end{array}$} & $\begin{array}{c}\text { Average \% } \\
\text { of Change }\end{array}$ & $\begin{array}{c}\text { \% of Simula- } \\
\text { tions }^{2,4}\end{array}$ \\
\hline \hline 1 & Assessment-based & 345000 & 2023454 & 2516 & 40974 & 44087 & $-68.2 \%$ & $99.8 \%$ \\
\hline 2 & Assessment-based & 345000 & 2023422 & 2533 & 118162 & 87095 & \\
\hline 3 & Fixed Schedule 1 & 0 & 2349738 & 223881 & 98164 & 101668 & $-9.4 \%$ & $70.0 \%$ \\
\hline 4 & Fixed Schedule 2 & 0 & 1998642 & 210306 & 300720 & 213582 & $225.6 \%$ & $0.7 \%$ \\
\hline 5 & Fixed Schedule 2 & 0 & 1998642 & 210306 & 297443 & 162746 & $261.7 \%$ & $1.6 \%$ \\
\hline
\end{tabular}

${ }^{1}$ Calculated using all 5000 iterations and all 100 years of each simulation (500 000 total values)

${ }^{2}$ Calculated using all 5000 iterations and the last 10 years of each simulation (50 000 total values)

${ }_{4}^{3}$ Average percentage of change in projected abundance of spawning-phase sea lamprey from that of the status quo (Variant 2)

${ }^{4}$ Percentage of simulations with a projected abundance of spawning-phase sea lamprey less than that of the status quo (Variant 2)

'Reducing larval assessment uncertainty had a much larger effect on forecasted abundance than reducing growth uncertainty. When we repeated the assessment-based strategy but assumed a lower level of assessment uncertainty, the average forecasted abundance was approximately one-third of the baseline value (Variants 1 and 2, Table 4), and the forecasted abundance for individual simulations was nearly always lower than the status quo variant. Conversely, repeating the Fixed Schedule 2 strategy, but assuming perfect knowledge of stream-specific larval growth rates had very little effect on the outcome (Variant 5, Table 4).

\section{DISCUSSION}

MSE-style models have been developed for several exploited fish stocks to simulate the entire management process and to examine the effects of various types of uncertainty in fishery management $[6,35,36]$. In this paper, we describe an MSE-style operating model for sea lamprey management, in which we have incorporated a) demographic (process) uncertainty - uncertainty in larval population dynamics, b) assessment uncertainty - imperfect knowledge used for stream selection, and c) control uncertainty - variable efficacy of chemical treatments. The model can be used to examine a variety of strategic questions concerning sea lamprey control in the Great Lakes by simulating the performance of the management system while explicitly accounting for these uncertainties.

We used the sea lamprey operating model to conduct a set of simulation experiments, forecasting the average number of spawning-phase sea lampreys in Lake Michigan resulting from five variants which differed in the management strategies used for stream selection and levels of two sources of uncertainty. At current levels of assessment uncertainty, reallocation of the funds used to assess stream reaches to support their treatment based on a fixed treatment schedule resulted in a lower expected abundance of spawning-phase sea lamprey in our simulations for Lake Michigan. We note that this strategy may not be the optimal fixed-schedule for the budget available; optimizing the schedule (beyond the scope of this analysis) might yield even better performance for the same budget. In contrast, treating stream reaches on a fixed schedule with no additional resources available for chemical treatments was much less effective than selecting reaches for treatment based on larval assessment, highlighting the sensitivity of the average performance of fixedschedule strategies to relatively small variations in budget. The most desirable outcomes were produced by the management variant with relatively low assessment uncertainty, but the costs associated with achieving this increased accuracy remain unknown.

These results raise important questions about the role of larval population assessment in sea lamprey control. We concluded that the current budget for treatment of Lake Michigan sea lamprey producing streams was only sufficient for successful control if the selection of streams was guided by an assessment program. On the other hand, comparatively small increases in funds for stream treatment (a 16\% increase from current levels) were sufficient to overcome the reduced accuracy of a fixed treatment schedule. Ultimately, our results suggest that alternative strategies where less is invested in assessment deserve consideration by sea lamprey program managers. In a related empirical study, the current assessment procedure that the operating model in this paper simulates was compared to an alternative rapid assessment method [37]. This rapid assessment method can be viewed as somewhere between the two extremes simulated here (current assessment versus no assessment), and was found to outperform the current method, but only if the savings from using the rapid assessment method were applied to control, similar to Variant 3 in our simulation experiment. The Great Lakes Fishery Commission adopted the rapid assessment method in 2008. While our results could be used to argue that a strategy with no assessment program at all is viable, the absence of any assessment would limit a manager's ability to verify the performance of the strategy and the operating model on which it is based.

Our results also highlight the particular importance of assessment uncertainty for sea lamprey management. When assessment uncertainty was reduced to the minimum level observed in Steeves [22], the assessment-based decision rule 
outperformed any other method of stream selection, despite the fact that more money was allocated to treatments under the Fixed Schedule 1 variant. Of course if assessment uncertainty were to be reduced by increasing annual assessment expenditures (i.e., more intensive population assessments), then the gains in suppression forecasted here would probably not be realized, at least absent an overall increase in the budget. However, it is possible that valuable reductions in assessment uncertainty could be achieved without increasing annual expenditures, particularly by improving the accuracy of models used to interpret assessment data. A noteworthy example of this concerns the model that is used to predict the proportion of the larval population in a stream reach that is expected to metamorphose during the year following an assessment [19]. Recent research into factors affecting sea lamprey metamorphosis has yielded a new model for forecasting metamorphosis for use in the stream ranking process that appears to be more accurate than the model currently in use [32]. Replacement of the current model with this newer model could substantially reduce the uncertainty associated with use of assessment data to rank streams for lampricide treatment.

For practical reasons all simulation tests of potential management strategies reflect choices regarding what uncertainties to address, and to what extent [10]. While we did explicitly incorporate some uncertainties, others were dealt with only through limited sensitivity analysis, and others were not quantitatively addressed. In principle Management Strategy Evaluation, to be comprehensive, should include a thorough evaluation of the robustness of the forecasted performance of each policy to all sources of uncertainty. In addition to the results for the base model presented in this manuscript, we also evaluated the same strategies using an alternative model for how sea lamprey grew. In that alternative model mean length-at-age followed a von Bertalanffy model, but the larval length distribution at each age followed a normal distribution, with no change to the distribution about the mean occurring to reflect the earlier transformation of faster growing larvae. This alternative version of the model produced very similar results to those reported here, suggesting that the specifics of among individual differences in growth are unlikely to have a strong influence on our results. We believe there remains scope for further sensitivity analysis of our sea lamprey MSE, particularly with respect to the highly influential larval survival parameter we used to calibrate our simulations.

As we have shown here, the sea lamprey operating model offers a valuable management tool for both simulating the impacts of management decisions and exploring the relative influences of various sources of uncertainty. Our simulation experiment demonstrated the large influence of assessment uncertainty on the effectiveness of management actions and the comparatively small influence of growth (process) uncertainty. In an earlier study [30], a simpler model was used to demonstrate the large influence of process uncertainty in recruitment on the relative performance of control strategies that either targeted larval populations (i.e., lampricide control) or targeted adult reproductive success (e.g., traps and sterile male releases). We have also used the operating model to compare the performance of different approaches to ranking streams for lampricide treatment based on assess- ment data [33], to compare control strategies that rely exclusively on chemical control of larval populations to those that utilize a mix of chemical control and methods that target adult reproductive success [31], and to determine management targets for sea lamprey control using Economic Injury Level [38] calculations [39]. Reporting the results of these applications is beyond the scope of this paper but will be the subject of future reports. Overall, we see great promise for the use of MSE-style approaches to inform fisheries management and, in this paper, present evidence that such analyses are beneficial even when management strategies are not directly focused on regulating harvest.

\section{ACKNOWLEDGEMENTS}

The research described in this paper was supported through funding from the Great Lakes Fishery Commission to MLJ and JRB, and from the Michigan Department of Natural Resources, Fisheries Division (in part through U.S. Fish and Wildlife Service Sport Fish Restoration Program Project F-80) to JRB. We are grateful for advice on model specification and parameter values from biologists with the U.S. Fish and Wildlife Service, Canada Department of Fisheries and Oceans, and the Great Lakes Fishery Commission, particularly members of the sea lamprey control program Assessment Task Force. We thank in particular Gavin Christie for his support of this work. This paper is contribution number 2009-09 of the Quantitative Fisheries Center at Michigan State University.

\section{Appendix A. Description of Alternative Larval Habitat Areas}

In addition to stream reaches targeted by lampricide control, the operating model represented three other areas considered capable of supporting larval sea lamprey. First, some lentic areas of the Great Lakes are known to contain larval sea lampreys (called "lentic habitats" here), presumably populated by downstream emigration of larvae from nearby streams where spawning occurred. These lentic habitats can be treated, for a cost, using granular Bayluscide. Second, there are areas that we presume to be untreatable, either because they have not yet been detected by surveys or are not cost-effective to treat ("untreated habitat"). Unlike the individual stream reaches in the model, however, these two types of areas are not intended to represent specific locations, but rather general habitat types that are capable of supporting larval sea lamprey. Third, a representation of the St. Marys River was included in the operating model for simulations of Lake Huron ("river habitat"; included as 0 habitat area for the currently presented simulations of Lake Michigan). For these alternative habitat representations, demographic parameters were based on averages from the stream-specific database for that lake. It has been suggested that growth rates of larvae in lentic habitats might generally be lower than in streams, but there are no empirical data to currently justify a different growth assumption for these habitats. Finally, we simulated population assessments for individual units of the lentic habitats using the same uncertainty assumptions as described for the stream reaches. There is no reason to expect larval density estimates for these habitats would differ in precision from those for stream habitat. 
The total area of lentic habitats for each Great Lake was estimated by sea lamprey biologists and managers (T.B. Steeves, M. Fodale, and J. Slade, unpublished data). We simulated the annual colonization of lentic habitats by assuming that a small fraction of the age- 0 production emigrates from all streams to lentic habitats. We adjusted this fraction during model calibration, such that the resulting average density of lentic-dwelling larvae was approximately $25 \%$ of the average larval density in streams, based on limited data on stream/lentic habitat density ratios for 11 Great Lakes streams (T.B. Steeves, DFO, unpublished data).

This approach to colonizing lentic habitats is clearly a simplification, in that not all streams actually contribute larvae to lentic habitats and there is likely emigration of larvae older than age- 0 . However, there are few if any data to support a more realistic representation at this time, and we felt that the key requirement was to connect lentic recruitment to stream production generally, rather than developing a spatially explicit model of stream-lentic connections. Further exploration of the significance of this simplification to our model may be warranted in the future, particularly if the treatment of lentic habitats becomes an important component of Great Lakes sea lamprey control.

Once age- 0 sea lampreys were allocated to the overall lentic habitat for a lake, they were distributed among individual lentic habitat units based on observations of the distribution of larvae among lentic-type habitats in the St. Marys River. We used data from 25 St. Marys River plots that had larval abundance estimates to define a relationship between the cumulative area of plots $(x)$ and the cumulative proportion of the total larval population $(y)$ in all plots and fitted the following function to these data:

$$
y=\frac{\left(1-e^{-\lambda x}\right)}{\left(1-e^{-\lambda}\right)}
$$

where $\lambda$ describes the extent to which the majority of the larvae are in a small proportion of the total area, with larger values implying a less uniform distribution among units.

The number of individual lentic habitat units $(N)$ was determined based on dividing the total lentic area for a lake by an average size calculated for 56 lentic treatment units (plots) in the St. Marys River that were considered for treatment during 2006 (12.5 ha; Gavin Christie, GLFC, unpublished data). During each year of a simulation, we distributed the age- 0 larvae allocated to treatable lentic habitat among the $N$ individual lentic units as:

$$
L_{i, 0, t}=\left\{\begin{array}{c}
\bar{L}_{0, t} \cdot \frac{\left(1-e^{-\lambda x_{i}}\right)}{\left(1-e^{-\lambda}\right)}, \text { if } i=1 \\
\bar{L}_{0, t} \frac{\left(1-e^{-\lambda x_{i}}\right)}{\left(1-e^{-\lambda}\right)}-\sum_{j=1}^{i-1} L_{j, 0, t}, \text { for } i=2 \text { to } N
\end{array}\right.
$$

where $\bar{L}_{0, t}$ is the total number of age- 0 larvae allocated to the lentic habitat for a given year $t$, and $x_{i}$ is the proportion of total lentic area in units 1 through $i$, and $\lambda$ was 3.02 based on the fit of equation A1. Once calculated, the proportion of larvae allocated to an individual lentic unit was held constant for all simulation years. Next, we assumed that the treatment costs for individual lentic units would be comparable to recent costs for actual treatment of St. Marys River plots (\$5 000 ha $^{-1}$; Gavin Christie, unpublished data). Thus, the simulated individual lentic units were of equal size and had equal treatment cost per area but varied in larval density.

The contribution of untreatable habitats to parasitic sea lamprey production in the Great Lakes is unknown. Based on the expert opinion of sea lamprey control program biologists and managers, we fixed the area of untreatable larval habitat at $2 \%$ of the total area of the total larval habitat in streams for each lake. We assumed, in effect, that larval densities in these untreatable habitats were comparable to those in stream larval habitats. These untreatable habitats contributed the same proportion of their age- 0 larval production to lentic habitats each year as was contributed from stream reaches.

\section{Appendix B. Parameterization of the von Bertalanffy Larval Growth Model for Sea Lamprey Based on Stream-Specific Growth Parameters Originally Devel- oped for a Linear Growth Model}

This appendix describes how the stream-specific parameters used in the von Bertalanffy model (equation 4) were obtained based on information already available in the ESTR database [19]. The stream-specific growth rates in ESTR were determined originally for use in a linear growth model. For each Great Lake, the previously assembled database included stream-specific estimates of both the duration of the growing season $\left(d_{i}\right)$, in days, and the average daily growth rate $\left(G_{i}\right)$, and had been used to predict mean length at age for stream $i$ based on:

$\bar{\ell}_{a, i}=a d_{i} G_{i}+\bar{\ell}_{0}$

where $a$ is age, and $\bar{\ell}_{0}$ is a user-specified length at age- 0 $(20 \mathrm{~mm})$. Recent information suggests that growth of larval sea lampreys is asymptotic [33]. Therefore, a von Bertalanffy growth model would likely provide a better representation of length at age for larval sea lamprey. Thus we describe a method by which existing stream-specific database values $\left(d_{i}\right.$ and $\left.G_{i}\right)$, derived for a linear growth model, could be used to specify stream-specific parameters in a non-linear growth model.

As with the linear growth model, the time available for growth can be represented by $a$ and $d_{i}$, which both can be directly incorporated into a nonlinear model. However, the linear-model form of the stream-specific daily growth rate $\left(G_{i}\right)$ cannot simply be inserted into the exponent of the nonlinear model. Therefore, we scaled the stream-specific values of $G_{i}$, recorded in the existing database, to serve as the Brody growth parameter $\left(\kappa_{i}\right)$ in the von Bertalanffy model using an assumption about a reference point. The selected reference point was the length at which $50 \%$ of larval sea lamprey would be expected to metamorphose $\left(\ell_{T}\right)$ and was 
specific to the transformation curve (specific to either the upper or lower Great Lakes).

By assuming that $\ell_{T}$ should be reached at the same age regardless of which growth model is chosen, we can solve for a relationship between $\kappa_{i}$ and $G_{i}$ :

$$
\kappa_{i}=\frac{-\ln \left(\frac{\ell_{T}-\bar{\ell}_{\infty}}{\bar{\ell}_{0}-\bar{\ell}_{\infty}}\right)}{\left(\ell_{T}-\bar{\ell}_{0}\right)} G_{i}
$$

such that $\kappa_{i}$ is directly proportional to $G_{i}$. This assumption suggests that the parameters for the original linear growth model were relevant because they produced length-at-age values which in turn produced a realistic pattern of transformation-at-age. In other words, both the linear model (equation B1) using $G_{i}$ and the von Bertalanffy model (equation

4) using $\kappa_{i}$ should produce $\ell_{T}$ at precisely the same age.

\section{REFERENCES}

[1] Harwood J, Stokes K. Coping with uncertainty in ecological advice: lessons from fisheries. Trends Ecol Evol 2003; 18: 617-22.

[2] Morgan MG, Henrion M. Uncertainty - A Guide to Dealing with Uncertainty in Quantitative Risk and Policy Analysis. Cambridge: Cambridge University Press 1990.

[3] Regan HM, Colyvan M, Burgman MA. A taxonomy and treatment of uncertainty for ecology and conservation biology. Ecol Appl 2002; 12: 618-28.

[4] Kirkwood GP. In: Pikitch EK, Huppert DD, Sissenwine MP, Eds. Global Trends: Fisheries Management. Bathesda, Mary land: American Fisheries Society Symposium 20 1997; pp. 41-99.

[5] Butterworth DS, Punt, AE. Experiences in the evaluation and implementation of management procedures. ICES J Mar Sci 1999; 56: 985-98.

[6] Punt AE. The FAO precautionary approach after almost 10 years: have we progressed towards implementing simulation-tested feedback-control management systems for fisheries management? Nat Res Mod 2006; 19: 441-64.

[7] Smith ADM, Sainsbury KJ, Stevens RA. Implementing effective fisheries-management systems - management strategy evaluation and the Australian partnership approach. ICES J Mar Sci 1999; 56: 967-79.

[8] Food and Agriculture Organization (FAO). Precautionary approach to fisheries, Part 2: Scientific papers. FAO Fish Tech Pap 1996; No. $350 / 2$

[9] Marasco RJ, Goodman D, Grimes CB, Lawson PW, Punt AE, Quinn TJ II. Ecosystem-based fisheries management: some practical suggestions. Can J Fish Aquat Sci 2007; 64: 928-39.

[10] Bence JR, Dorn MW, Irwin BJ, Punt AE. Recent advances in the evaluation and implementation of harvest polices. Fish Res 2008; 94: 207-9.

[11] Peters CN, Marmorek DR, Deriso RB. Application of decision analysis to evaluate recovery actions for threatened Snake River fall Chinook salmon (Oncorhynchus tshawytscha). Can J Fish Aquat Sci 2001; 58: 2447-58.

[12] Alexander CAD, Peters CN, Marmorek DR, Higgens P. A decision analysis of flow management experiments for Columbia River mountain whitefish (Prosopium williamsoni) management. Can J Fish Aquat Sci 2006; 63: 1142-56.

[13] Haeseker SL, Jones ML, Peterman RM, Bence JR, Dai W, Christie GC. Explicit consideration of uncertainty in Great Lakes fisheries management: decision analysis of sea lamprey (Petromyzon marinus) control in the St. Marys River. Can J Fish Aquat Sci 2007; 64: 1456-68.
[14] Smith BR, Tibbles JJ. Sea lamprey (Petromyzon marinus) in Lakes Huron, Michigan, and Superior: history of invasion and control, 1936-78. Can J Fish Aquat Sci 1980; 37: 1780-1.

[15] Brege DC, Davis DM, Genovese JH, McAuley TC, Stephens BE, Westman RW. Factors responsible for the reduction in quantity of the lampricide, TFM, applied annually in streams tributary to the Great Lakes form 1979 to 1999. J Great Lakes Res 2003; 29 (Suppl 1) : 500-9.

[16] Christie GC, Goddard CI. Sea lamprey international symposium (SLIS II): advances in the integrated management of sea lamprey in the Great Lakes. J Great Lakes Res 2003; 29 (Suppl 1): 1-14.

[17] Lavis DS, Henson MP, Johnson DA, Koon EM, Ollila DJ. A case history of sea lamprey control in Lake Michigan: 1979-1999. J Great Lakes Res 2003; 29 (Suppl 1): 584-98.

[18] Twohey MB, Heinrich JW, Seelye JG, et al. The sterile-malerelease technique in Great Lakes sea lamprey management. J Great Lakes Res 2003; 29 (Suppl 1): 410-23.

[19] Christie GC, Adams JV, Steeves TB, et al. Selecting Great Lakes streams for lampricide treatment based on larval sea lamprey surveys. J Great Lakes Res 2003; 29 (Suppl 1): 152-60.

[20] Slade JW, Adams JV, Christie GC, et al. Techniques and methods for estimating abundance of larval and metamorphosed sea lampreys in Great Lakes tributaries, 1995 to 2001. J Great Lakes Res 2003; 29 (Suppl 1): 137-51.

[21] Heimbuch DG, Youngs WD. Application of decision analysis to sea lamprey control. Great Lakes Fishery Commission Project Completion Report. Ann Arbor, MI 1982.

[22] Steeves TB. Uncertainty in estimating sea lamprey (Petromyzon marinus) abundance in Great Lakes tributaries. Masters Thesis, Michigan State University, East Lansing, MI 2002.

[23] Hansen MJ, Adams JV, Cuddy DW, et al. Optimizing larval assessment to support sea lamprey control in the Great Lakes. J Great Lakes Res 2003; 29 (Suppl 1): 766-82.

[24] Bence JR, Bergstedt RA, Christie GC, et al. Sea Lamprey (Petromyzon marinus) parasite-host interactions in the Great Lakes. J Great Lakes Res 2003; 29 (Suppl 1): 253-82.

[25] Hansen GJA, Jones ML. The value of information in fishery management. Fisheries 2008; 33: 340-48.

[26] Bergstedt RA, Seelye JG. Evidence for lack of homing by sea lampreys. Trans Am Fish Soc 1995; 124: 235-39.

[27] Morman RH, Cuddy DW, Rugen PC. Factors influencing the distribution of sea lamprey (Petromyzon marinus) in the Great Lakes. Can J Fish Aquat Sci 1980; 37: 1811-26.

[28] Mullett KM, Heinrich JW, Adams JV, et al. Estimating lake-wide abundance of spawning-phase sea lampreys (Petromyzon marinus) in the Great Lakes: extrapolating from sampled streams using regression models. J Great Lakes Res 2003; 29 (Suppl 1): 240-52.

[29] Sorensen PW, Vrieze LA. The chemical ecology and potential application of the sea lamprey migratory pheromone. J Great Lakes Res 2003; 29 (Suppl 1): 66-84.

[30] Jones ML, Bergstedt RA, Twohey MB, Fodale MF, Cuddy DW, Slade JW. Compensatory mechanisms in Great Lakes sea lamprey populations: implications for alternative controls. J Great Lakes Res 2003; 29 (Suppl 1): 113-29.

[31] Dawson HA. Recruitment Dynamics of Great Lakes Sea Lamprey (Petromyzon marinus) Populations and Implications for Integrated Pest Management. Ph.D. Dissertation, Michigan State University, East Lansing, MI 2007.

[32] Treble AJ, Jones ML, Steeves TB. Development and evaluation of a new predictive model for metamorphosis of Great Lakes larval sea lamprey (Petromyzon marinus) populations. J Great Lakes Res 2008; 34: 404-17.

[33] Treble AJ. Choosing Streams for Sea Lamprey Control: Using Alternative Models Of Metamorphosis to Optimize the Stream Selection Process. Masters Thesis, Michigan State University, East Lansing, MI 2006.

[34] Szalai EB, Bence JR, Jones ML. Estimating sea lamprey damage to fish populations in Lakes Michigan and Huron. Great Lakes Fishery Commission Completion Report 2005.

[35] Punt AE, Smith ADM. Harvest strategy evaluation for the eastern stock of gemfish (Rexea solandri). ICES J Marine Sci 1999; 56: 860-75.

[36] Dichmont CM, Deng A, Punt AE, Venables W, Haddon M. Management strategies for short-lived species: The case of Australia's Northern Prawn Fishery 1. Accounting for multiple species, spatial 
structure and implementation uncertainty when evaluating risk. Fish Res 2006; 82: 204-20.

[37] Hansen GJA, Jones ML. A rapid assessment approach to prioritizing streams for control of Great Lakes sea lampreys: a case study in adaptive management. Can J Fish Aquat Sci 2008; 65: 2471-84.

[38] Koonce JF, Eshenroder RL, Christie GC. An economic injury level approach to establishing the intensity of sea lamprey control in the Great Lakes. N Am J Fish Manag 1993; 13: 1-14.
[39] Irwin BJ, Bence JR, Jones ML, Liu W. Defining targets for sea lamprey control in the Great Lakes: economic injury levels and fish community goal-based targets. Great Lakes Fishery Commission 2007 Project Completion Report and Quantitative Fisheries Center Technical Report 2008.

Received: October 10, 2008

(C) Jones et al.; Licensee Bentham Open.

This is an open access article licensed under the terms of the Creative Commons Attribution Non-Commercial License (http://creativecommons.org/licenses/by-nc/3.0/) which permits unrestricted, non-commercial use, distribution and reproduction in any medium, provided the work is properly cited. 